\title{
Identification of Hub Genes and Key Pathways Associated With Bipolar Disorder Based on Weighted Gene Co-expression Network Analysis
}

\author{
Yang Liu' ${ }^{1}$, Hui-Yun Gu${ }^{2}$, Jie Zhu ${ }^{3}$, Yu-Ming Niu', Chao Zhang ${ }^{1 *}$ and Guang-Ling Guo ${ }^{4 *}$ \\ ${ }^{1}$ Center for Evidence-Based Medicine and Clinical Research, Taihe Hospital, Hubei University of Medicine, Shiyan, China, \\ ${ }^{2}$ Department of Orthopedic, Zhongnan Hospital of Wuhan University, Wuhan, China, ${ }^{3}$ Trade Union, Taihe Hospital, Hubei \\ University of Medicine, Shiyan, China, ${ }^{4}$ Center of Women's Health Sciences, Taihe Hospital, Hubei University of Medicine, \\ Shiyan, China
}

OPEN ACCESS

Edited by:

Luis Mendoza,

National Autonomous University

of Mexico, Mexico

Reviewed by:

Shilpa Dhar,

University of Texas MD Anderson

Cancer Center, United States

Xian-Tao Zeng,

Wuhan University, China

Carlos Alberto Moreira-Filho,

University of São Paulo, Brazil

*Correspondence:

Chao Zhang

zhangchao0803@126.com

Guang-Ling Guo

guoguangling1208@163.com

Specialty section:

This article was submitted to

Systems Biology,

a section of the journal

Frontiers in Physiology

Received: 14 February 2019 Accepted: 07 August 2019

Published: 20 August 2019

Citation:

Liu Y, Gu H-Y, Zhu J, Niu Y-M,

Zhang C and Guo G-L (2019)

Identification of Hub Genes and Key Pathways Associated With Bipolar Disorder Based on Weighted Gene

Co-expression Network Analysis.

Front. Physiol. 10:1081

doi: 10.3389/fphys.2019.01081
Bipolar disorder (BD) is a complex mental disorder with high mortality and disability rates worldwide; however, research on its pathogenesis and diagnostic methods remains limited. This study aimed to elucidate potential candidate hub genes and key pathways related to $\mathrm{BD}$ in a pre-frontal cortex sample. Raw gene expression profile files of GSE53987, including 36 samples, were obtained from the gene expression omnibus (GEO) database. After data pre-processing, 10,094 genes were selected for weighted gene co-expression network analysis (WGCNA). After dividing highly related genes into 19 modules, we found that the pink, midnight blue, and brown modules were highly correlated with BD. Functional annotation and pathway enrichment analysis for modules, which indicated some key pathways, were conducted based on the Enrichr database. One of the most remarkable significant pathways is the Hippo signaling pathway and its positive transcriptional regulation. Finally, 30 hub genes were identified in three modules. Hub genes with a high degree of connectivity in the PPI network are significantly enriched in positive regulation of transcription. In addition, the hub genes were validated based on another dataset (GSE12649). Taken together, the identification of these 30 hub genes and enrichment pathways might have important clinical implications for BD treatment and diagnosis.

Keywords: bipolar disorder, hub genes, WGCNA, pre-frontal cortex, modules

\section{INTRODUCTION}

Bipolar disorder (BD), like other mental illnesses, is considered a disease caused by abnormal development of the nervous system. During the neural development of patients, the ganglion birth and death rates increased (Uribe and Wix, 2012). According to the world health organization (WHO) statistics on $\mathrm{BD}$, it is the sixth leading cause of disability worldwide.

The primary symptom of $\mathrm{BD}$ is a pathological increase or decrease in emotional activity, and the pathological process is a recurrent episode of mania and depression. The characteristics of mania are high mood, marked acceleration of thinking, and increased verbal movements. Conversely, depression often leads to low mood, slow thinking, reduced speech and movement, and loss of 
appetite (Cox et al., 2014). Thus, the alternation of these two diseases can significantly impact the health of the patient. BD can cause circadian rhythm disturbances (Gonzalez, 2014), cognitive impairment, verbal memory loss (Cardoso et al., 2015), and increase the risk of other diseases. Suicide (Calkin and Alda, 2012) is the leading cause of death in BD patients. In one longterm study, the average suicide rate of major emotional patients was $15-20 \%$, and while BD patients had a rate of more than $20 \%$ (Pompili et al., 2013). Furthermore, the suicide of a patient can greatly affect their family and society.

Bipolar disorder has a high rate of misdiagnosis, prevalence and mortality. As the clinical manifestations of unipolar and bipolar depression are difficult to distinguish, and unipolar depression is more common (Hirschfeld, 2014). Therefore, it is crucial to find the hub genes that influence $\mathrm{BD}$ to improve the current treatment status. The prefrontal cortex is often called the center of the command and emotional control of the brain and is inextricably linked to mental illness. Microarray analysis of gene expression profiles in the prefrontal cortices from $\mathrm{BD}$ patients might contribute to the identification of hub genes and key pathways related to BD supporting the development of a new treatment strategy.

Weighted gene co-expression network analysis (WGCNA), which has been proposed by scholars Zhang and Horvath (2005), is a highly efficient and accurate biological methodology for Microarray data. The WGCNA package was released in 2008 (Langfelder and Horvath, 2008). To date, related studies have preformed gene expression microarray profiling of diseases to identify the hub genes related to diseases based on WGCNA, including osteoporosis (Farber, 2010), hepatocellular carcinoma (Yin et al., 2018), osteosarcoma (Liu et al., 2017), and renal cell carcinoma (Chen et al., 2018).

Weighted gene co-expression network analysis has been used to analyze large gene data sets. Build a matrix of all the genes and soft threshold of the data set is performed, and then the scalefree network is established through the soft threshold (Langfelder and Horvath, 2008). Tens of thousands of genes are divided into different modules in scale-free networks, and the genes in each module have the same expression pattern. After correlating these modules with the phenotypic characteristics of the sample, modules with a high correlation with the sample features are selected. Finally, hub genes with high connectivity in the module were identified. These genes play key roles in the characteristics and the development of the disease. Thus, the aim of the present study was to find the hub genes, key pathways, and potential molecular mechanisms of BD.

\section{MATERIALS AND METHODS}

\section{Data Collection and Pre-processing}

The gene expression microarray profile of $\mathrm{BD}$ was obtained from the gene expression omnibus (GEO) database ${ }^{1}$ (Barrett et al., 2013). There were 205 samples in the data set of GSE53987 (Lanz et al., 2019), including 48 patients with schizophrenia, 52

${ }^{1}$ http://www.ncbi.nlm.nih.gov/geo patients with $\mathrm{BD}, 50$ patients with major depressive disorder, and 55 healthy individuals. Each state is divided into the prefrontal cortex, striatum, and hippocampus, depending on the tissue source. In this study, we selected 36 samples, including $17 \mathrm{BD}$ and 19 healthy controls (HC). All samples were derived from Postmortem human sample's pre-frontal cortex, and the $\mathrm{BD}$ group was indistinguishable from the $\mathrm{HC}$ group in mean age, postmortem interval, brain $\mathrm{pH}$, RNA integrity number (RIN). All tissue samples were based on the platform of the Affymetrix Human Genome U133 Plus 2.0 Array. Robust multiarray averaging (RMA) algorithm was used to process the original file to ensure the comparability of gene expression profiles (Irizarry et al., 2003). The nsFilter algorithm was used to remove the probes that had little variation in the expression value, as well as the probes that did not have a corresponding gene ID (Yin et al., 2018).

\section{Co-expression Network Construction}

Weighted gene co-expression network analysis package was necessary for the co-expression network construction (Langfelder and Horvath, 2008). First, the function goodSamplesgenes was used to remove unqualified genes and samples, and Z.k $<-2.5$ was excluded from subsequent studies. Then, function pickSoftThreshold was used to choose an appropriate soft-thresholding power $(\beta)$ based on a scalefree topology criterion. The weighted adjacency matrix was constructed using the soft-thresholding power. The relationship between one gene and all other ones in the analysis was incorporated, and the adjacency matrix was transformed into the topological matrix (TOM) (Yip and Horvath, 2007). The genes demonstrated hierarchical clustering using the flashClust function, according to the TOM-based dissimilarity (1-TOM) measure. After hierarchical clustering, highly interconnected genes were assigned to the same module (Ravasz et al., 2002).

\section{Identification of Clinically Significant Modules and Functional Annotation}

After the effective clinical information was imported into the co-expression network, the module eigengene (ME), gene significance (GS), and module membership (MM) were calculated. ME was representative of the gene expression profiles in a module, representing the average expression level of genes in this module. MM was defined as the degree of correlation between genes and module. If MM is close to 0 , then the gene is not part of the module. Conversely, if MM is close to 1 or -1 , the gene is highly correlated with the module. GS can be considered to be the association of individual genes with clinical information. The module could be a candidate if it has a high ME valve with the clinical trait (Langfelder and Horvath, 2008). The annotating the function of relevant modules was preformed to the potential mechanisms for the effects of corresponding clinical symptoms. All genes in the candidate modules were uploaded to the Enrichr database (Kuleshov et al., 2016) for pathway enrichment based on gene ontology (GO) functional annotation and the kyoto encyclopedia of genes and genomes (KEGG). GO enrichment 
analysis is primarily divided into three parts, molecular function (MF), biological process (BP), and cellular component (CC).

\section{Identification and Validation of Hub Gene}

Hub genes are defined as genes with high correlation in candidate modules. High connectivity means that the connectivity ranked at top $10 \%$. For example, if the module size was 1000 , then the genes with top 100 were defined as the hub genes. Moreover, the hub gene has to meet the absolute value of the geneModuleMembership $>0.80$ and geneTraitSignificance $>0.20$. After identifying hub genes highly associated with clinical traits, search tool for the retrieval of interacting genes (STRING) (Szklarczyk et al., 2015) database was used to construct a PPI network and Cytoscape and (Shannon et al., 2003) visualize the PPI network. If a gene has high degrees in a PPI network, it will be defined as playing a critical role in the module. At last, hub genes selected will be validated. A separate dataset GSE12649 (Iwamoto et al., 2005) was used to verify the differential in the expression of hub genes in clinical traits using one-way ANOVA $(P<0.05)$. In order to prevent the gender and age of the sample having an impact on the selected hub genes, we used one-way covariance analysis to detect it.

\section{RESULTS}

\section{Data Pre-processing and Co-expression Network Construction}

We downloaded 36 raw files from BD patients' pre-frontal cortex from the GEO database. A total of 10094 genes were obtained from 36 samples through the RMA algorithm and nsFilter. In subsequent studies, samples GSM1304927 and GSM1304952 were filtered out (Supplementary Figure 1). On the basis of the scale-free topology criterion, to define the adjacency matrix, we selected $\beta=6$ which the scale-free topology fit index reaches 0.88 as the soft-thresholding power (Supplementary Figure 2). As shown in Supplementary Figure 3, the 10,094 genes were divided into 19 modules based on the dynamic tree cutting method.

\section{Identification of Clinically Significant Modules and Functional Annotation}

After the clinical traits of the samples were introduced into the weighted network (Figure 1), The study found Pink $(r=0.51$, $P=0.002)$ module with 221 genes, brown $(r=0.42, P=0.01)$ with 1104 genes, and midnightblue module $(r=-0.41, P=0.02)$ with 138 genes were highly correlated with the disease. In the following analysis, the GS and MM of the midnight blue module (cor $=0.51$, $P=1.7 \mathrm{e}-10$, Figure 2), the brown module (cor $=0.44, P=1.3 \mathrm{e}-53$, Figure 2) and the pink module (cor $=0.48, P=4.8 \mathrm{e}-16$, Figure 2) were calculated. In order to clarify the functional mechanism of targeted modules and diseases, 143 hub genes were uploaded to the Enrichr database for functional annotation. Supplementary Table 1 shows clear information about 143 hub genes. GO function annotation indicates that hub genes were enriched in eye development, G-protein coupled receptor complex, positive regulation of transcription, negative regulation of canonical
Wnt signaling pathway, cytokine receptor activity complex, activating transcription factor binding, phosphatidylinositol binding, and others $(P<0.01)$ (Supplementary Table 2). Supplementary Table 3 shows that hub genes were enriched in the Hippo signaling pathway, thyroid hormone signaling pathway, signaling pathways regulating stem cell pluripotency, endocytosis, adherens junction, and others, based on KEGG pathway analysis $(P<0.01)$.

\section{Validation of Hub Gene and PPI Network Construction}

We use the methods mentioned above to identify hub genes. 143 hub genes in the three modules were identified. Figure 3 shows the PPI network for modules which are a candidate. The module network contained 96 nodes and 160 edges, three of which were identified as the high degree genes. Moreover, we found that hub genes with better connectivity in the PPI network are significantly enriched in the positive regulation of transcription (DNA-templated), including SOX2, FGF2, SMAD2, YAP1, PAX6, AGT, and FOXO1. This enrichment pathway might be a potential BD mechanism. All hub genes were validated based on GSE12649. Finally, we identified 30 hub genes common to both data sets as our final hub genes. Boxplots were used to show the validation results for 30 hub genes (Figure 4). Table 1 shows final hub genes in the three modules, and the covariate analyses of final hub genes are described in Supplementary Table 4.

\section{DISCUSSION}

Bipolar disorder, a disease that is spread worldwide, has high rates of disability (Vos et al., 2012), mortality, and suicide, which can impose a significant burden on society (Adam, 2008). Therefore, exploring the hub genes and key pathways related to $\mathrm{BD}$ is essential for the diagnosis and treatment of patients with BD.

The weighted gene co-expression network analysis algorithm searches for effective information from gene expression microarray profile by building gene modules, and it tries to explain the significance of a gene module from the perspective of biology. In this study, we found that the pink, midnightblue, and brown modules were highly correlated with $\mathrm{BD}$ or no-BD. The expression of 30 genes in these three modules showed significant changes in patients with $\mathrm{BD}$ and non-BD individuals, 25 of which were validated through one-way ANOVA. Although other five genes have not been validated, most of them have been mentioned in previous studies (Meira-Lima et al., 2000; Medina et al., 2013; Ferensztajn-Rochowiak et al., 2016). These hub genes may have important clinical significance in the course of BD patient diagnosis and prognosis. After covariate analysis of all hub genes, we found that gender had little effect on these genes $(P>0.05)$. A few hub genes are affected by age factors $(P>0.05)$, including SCD4, PDLIM5, FAM189A2, and MAOA.

Kyoto encyclopedia of genes and genomes enrichment analysis suggested that the hub gene is mainly enriched in the Hippo signaling pathway, thyroid hormone signaling pathway, adherens junction, endocytosis, cell adhesion molecule, and mineral absorption. Meanwhile, we found that SMAD2 (SMAD 

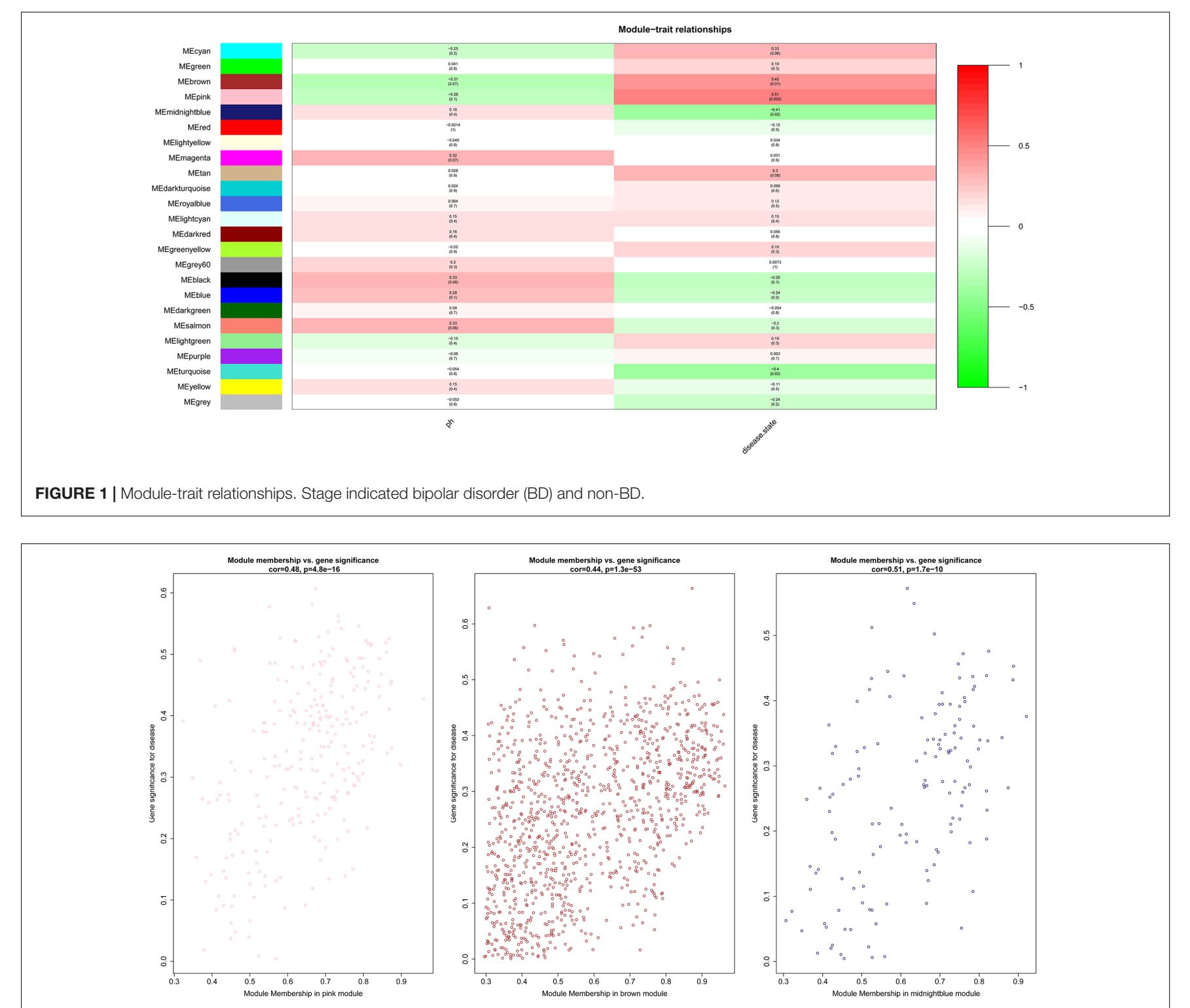

FIGURE 2 | Scatter diagram for module membership vs. gene significance of stage (BD or non-BD) in three modules.

family member 2), a member of the SMAD family, appears in most enrichment pathways. SMAD proteins are signal transducers and transcriptional modulators that mediate multiple signaling pathways. SMAD proteins regulate the signaling of transforming growth factor (TGF)-beta, and it plays a vital role in the growth, repair, and rotation of the nervous system (Liang et al., 2008).

The primary role of the Hippo signaling pathway is to regulate cell growth and apoptosis, maintaining organ size (Zhang et al., 2009). Moreover, it has another role in the establishment and maintenance of dendritic fields, since abnormalities in the Hippo signaling pathway can cause defects in these functions (Emoto, 2012). There is a significant difference in the dendritic structure between $\mathrm{BD}$ patients and normal individuals (Konopaske et al., 2014). The hub gene YAP1 (Yes-associated protein 1) is the main effector downstream of the Hippo signal pathway (Mizuno et al., 2012). Abnormalities in YAP1 expression will affect the function of the Hippo signal pathway. Also, YAP1 plays an essential role in the maintenance of neural progenitor cells (Mizuno et al., 2012). Neural progenitor cells regulate the production of the cerebral cortex (Arai and Taverna, 2017). The hub gene PARD3 (par3 family cell polarity regulator) and Hippo signaling pathway together maintain ordered RGP (radial glial progenitor) splitting behavior and neuron generation in the mammalian cortex, and asymmetric division of RGPs regulates the generation of cortical neurons (Kodaka and Hata, 2015; Liu et al., 2018). Dysfunction of this mechanism often leads to cortical neuron damage and may lead to the development of BD. Moreover, a recent study also mentioned that $\mathrm{BD}$ is associated with the Hippo signaling pathway (O’Connell et al., 2018). 
TABLE 1 | Hub genes from the interesting modules.

\begin{tabular}{|c|c|c|c|c|c|}
\hline Gene symbol & ENTREZ_GENE_ID & Gene ontology & geneModuleMembership & Gene trait significance & Up or down \\
\hline AGT & 183 & Alkylguanine DNA binding & 0.887157454 & 0.298842057 & Down \\
\hline AHCYL1 & 10768 & Adenosylmethionine hydrolase activity & 0.892921034 & 0.263587391 & Down \\
\hline APOE & 348 & Cognition & 0.906508824 & 0.259473598 & Down \\
\hline ATP1A2 & 477 & $\begin{array}{l}\text { Temperature gated cation channel } \\
\text { activity }\end{array}$ & 0.944732073 & 0.358469425 & Down \\
\hline CHRDL1 & 91851 & $\begin{array}{l}\text { Negative regulation of secondary } \\
\text { growth }\end{array}$ & 0.863182953 & 0.462817741 & Down \\
\hline EMX2 & 2018 & Forebrain neuroblast differentiation & 0.943545349 & 0.339285953 & Down \\
\hline ETNPPL & 64850 & / & 0.905878301 & 0.312454124 & Down \\
\hline FAM189A2 & 9413 & Gene expression & 0.872771128 & 0.313792924 & Down \\
\hline FGF2 & 2247 & fibroblast proliferation & 0.9348346 & 0.45704696 & Down \\
\hline GJA1 & 2697 & Gap junction & 0.920113458 & 0.328482543 & Down \\
\hline GNA12 & 2768 & Spermatid nucleus differentiation & 0.867055073 & 0.311316484 & Down \\
\hline HEPH & 9843 & Copper exporting atpase activity & 0.959262811 & 0.384839787 & Down \\
\hline IL17RB & 55540 & Cardiac fibroblast cell fate commitment & 0.87323481 & 0.315545635 & Down \\
\hline MAOA & 4128 & Monoamine oxidase activity & 0.889016805 & 0.495684 & Down \\
\hline METTL7A & 25840 & $\begin{array}{l}\text { RNA polymerase II regulatory region } \\
\text { DNA binding }\end{array}$ & 0.93466073 & 0.322509631 & Down \\
\hline $\mathrm{NOTCH} 2$ & 4853 & Notch signaling pathway & 0.952637565 & 0.360026782 & Down \\
\hline NTRK2 & 4915 & $\begin{array}{l}\text { Regulation of neurotrophin TRK } \\
\text { receptor signaling pathway }\end{array}$ & 0.947485184 & 0.327326464 & Down \\
\hline PARD3 & 56288 & $\begin{array}{l}\text { Positive regulation of JAK STAT } \\
\text { cascade }\end{array}$ & 0.877332438 & 0.357811988 & Down \\
\hline PAX6 & 5080 & Eye development & 0.912624234 & 0.408751454 & Down \\
\hline PDLIM5 & 10611 & Activation of bipolar cell growth & 0.92796358 & 0.456391993 & Down \\
\hline PPAP2B & 8613 & $\begin{array}{l}\text { Cell migration involved in coronary } \\
\text { angiogenesis }\end{array}$ & 0.882924502 & 0.299775962 & Down \\
\hline RAB31 & 11031 & ER body & 0.908780797 & 0.376400336 & Down \\
\hline SDC2 & 6383 & $\begin{array}{l}\text { Positive regulation of synapse } \\
\text { maturation }\end{array}$ & 0.935916785 & 0.333198546 & Down \\
\hline SDC4 & 6385 & Small molecule catabolic process & 0.889206651 & 0.361762932 & Down \\
\hline SLC1A3 & 6507 & $\begin{array}{l}\text { Negative regulation of } L \text { glutamate } \\
\text { transport }\end{array}$ & 0.930712467 & 0.322178332 & Down \\
\hline SMAD2 & 4087 & SMAD2 protein complex & 0.841744744 & 0.431128371 & Down \\
\hline SOX2 & 6657 & Stem cell differentiation & 0.960030288 & 0.437884345 & Down \\
\hline TP53BP2 & 7159 & P53 binding & 0.916365941 & 0.261334186 & Down \\
\hline YAP1 & 10413 & Hippo signaling & 0.946460117 & 0.317467764 & Down \\
\hline YES1 & 7525 & $\begin{array}{l}\text { Regulation of B cell antigen processing } \\
\text { and presentation }\end{array}$ & 0.913582422 & 0.40331199 & Down \\
\hline
\end{tabular}

Gene ontology function annotation indicates that hub genes are significantly represented in some biological processes such as eye development, G-protein coupled receptor complex, positive regulation of transcription, negative regulation of canonical Wnt signaling pathway, cytokine receptor activity, and activating transcription factor binding. The Wnt signaling pathway contributes to the development of neural circuits and neuronal plasticity as well as early neural induction (Kiecker and Niehrs, 2001; Stern, 2001; Ciani and Salinas, 2005). Neurodevelopmental disorders are often one of the main pathological features of mental illness. There is already a large body of evidence confirming that abnormalities in the Wnt signaling pathway contribute to BD pathogenesis (Mulligan and Cheyette, 2017). We have obtained many biological processes through GO enrichment analysis, and although many of them have not been mentioned, these may be potential mechanisms underlying the development of BD.

For the PPI network, we found significant connectivity between the three genes, including SOX2 (SRY-box 2), FGF2 (fibroblast growth factor 2), and APOE (apolipoprotein E). SOX2 is a transcription factor that regulates stem cell proliferation and differentiation in the central nervous system (Phi et al., 2007; Koike et al., 2014). Moreover, the SOX2-encoded product SOX2ot is considered to be a potential marker of neurodegenerative diseases (Arisi et al., 2011). FGF2 plays a crucial role in maintaining differentiation and function of the central nervous system (Woodbury and Ikezu, 2014), and the level of FGF2 in patients with $\mathrm{BD}$ is higher than that in healthy people (Liu et al., 2014). NOTCH2 (notch receptor 2) plays a key role in the development of the nervous 


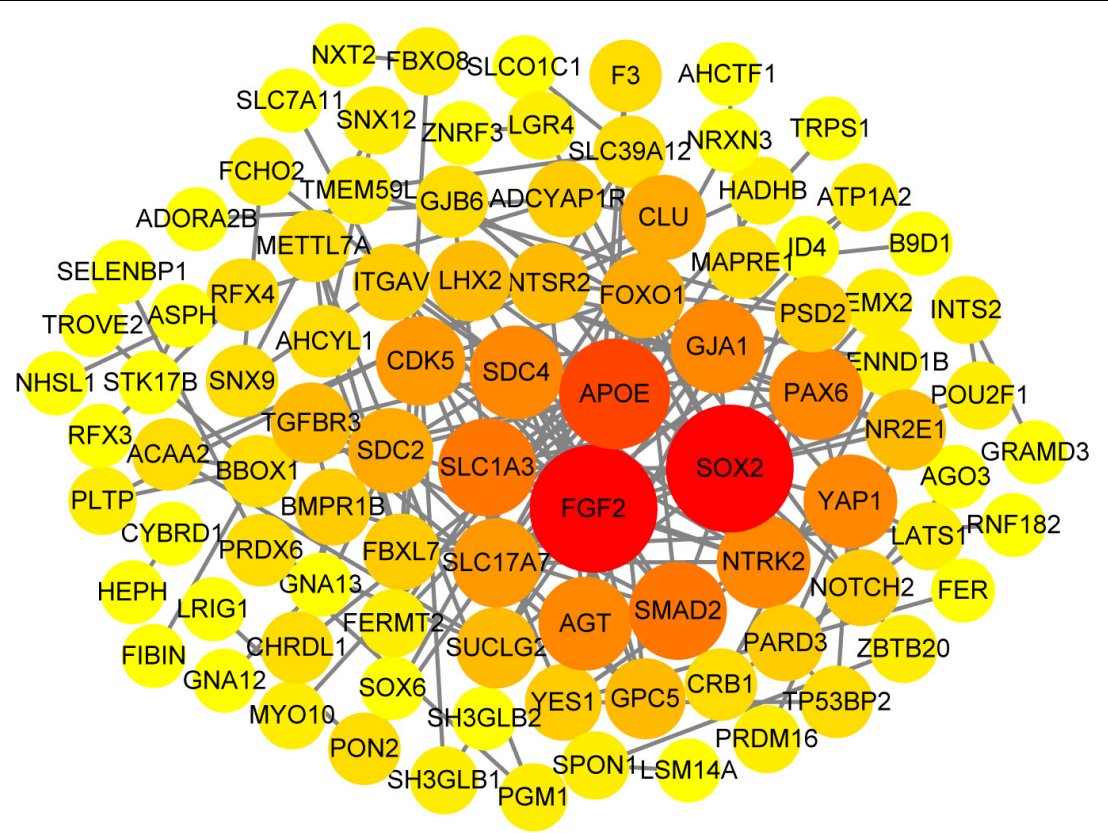

14

FIGURE 3 | PPI network for all candidate modules.

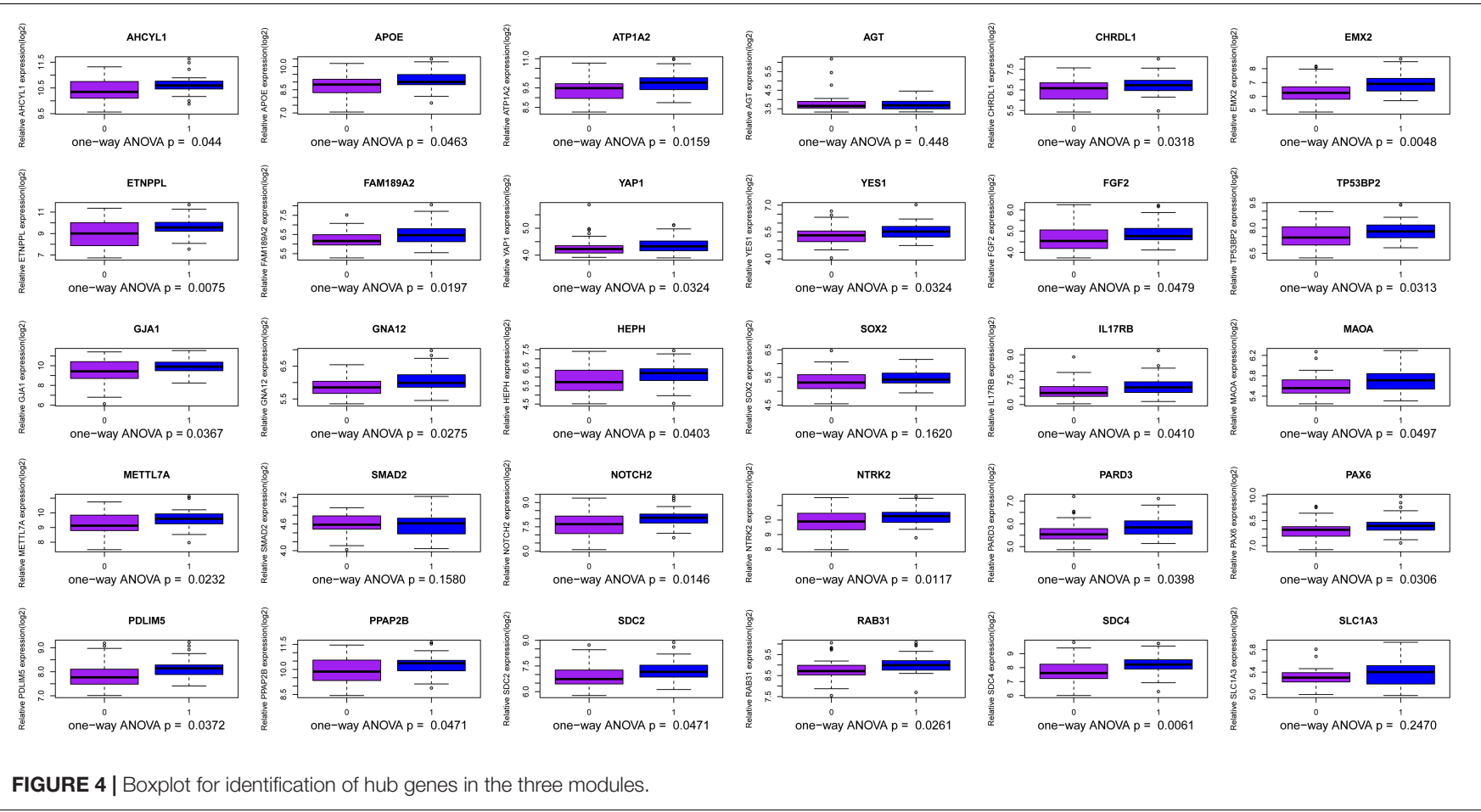

system (Sundararajan et al., 2018). A series of pathological and behavioral changes in $\mathrm{BD}$ patients may indicate that the development of the nervous system has been destroyed (O'Shea and McInnis, 2016). FGF2 can inhibit neuronal differentiation through the NOTCH pathway (Faux et al., 2001), which may affect the normal development of the nervous system, leading to BD. APOE apolipoprotein plays a key role in maintaining cerebral phospholipid homeostasis (Zhu et al., 2015) and plays a vital role in the pathogenesis and pathology of Alzheimer's disease (AD) (Raber et al., 2004). SLC1A3 (solute carrier family 
1 member 3) plays a role in the termination of excitatory neurotransmission in the central nervous system. SLC1A3 has been shown to decrease in BD patients (Medina et al., 2013). NTRK2 (neurotrophic receptor tyrosine kinase 2) is thought to have the effect of a mood stabilizer, which may affect the treatment of BD (Fabbri and Serretti, 2016). It is a receptor for neurotrophin brain-derived neurotrophic factor (BDNF). BDNF has nutritional and protective effects on nerves and is a potential biomarker for BD (Shaltiel et al., 2007; Munkholm et al., 2016). NTRK2 is considered a candidate gene for BD (Smith et al., 2009). AGT (angiotensinogen) was found to be associated with increased susceptibility to BD in Brazil (Meira-Lima et al., 2000).

Coincidentally, many genes with high connectivity in the PPI network were enriched in the positive regulation of transcription (DNA-templated), including SOX2, FGF2, SMAD2, YAP1, PAX6, AGT, and FOXO1. In a recent study, BD IPSC (induced pluripotent stem cells) is significantly different from normal IPSC in gene expression profiling, especially membrane receptor and ion channel genes, and calcium ion-related transcripts (Chen et al., 2014). It is well known that miRNA is one of the products of the transcription process, and miRNA expression level is closely related to transcriptional regulation. The expression levels of multiple MIRNAs in the prefrontal cortex of BD patients have changed (Kim A.H. et al., 2010; Moreau et al., 2011). The expression profile of astrocytes in the cerebral cortex of patients with $\mathrm{BD}$ has also increased (Toker et al., 2018). The activity of the transcription factor plays an essential role in neuronal function. Abnormal gene expression of transcription factors can affect the development and function of the nervous system and may lead to mental diseases, including BD (Pinacho et al., 2015).

Gap junction protein alpha 1 (GJA1) is a member of the connexin gene family, encodes connexin $(\mathrm{Cx})$ proteins, $\mathrm{Cx} 43$ (De Bock et al., 2013). GJA1 is widely expressed in astrocytes, mice lacking GJA1 are grossly normal, but their synaptic plasticity is reduced (Han et al., 2014). The development of BD also leads to changes in synaptic plasticity (Berridge, 2014), and BD pathology is associated with apoptosis and synaptic dysfunction (Kim H.W. et al., 2010). Moreover, the traditional therapeutic drug lithium agent of BD has a potential effect on the activation of neural plastic channels during the treatment process (MachadoVieira, 2018). However, there is currently no evidence that BD is associated with GJA1.

Both Bipolar disorder and Alzheimer's disease are neurologically related diseases, one is neurodevelopmental disorder (Sanches et al., 2008), and the other is neurodegeneration (Hogh, 2017). But they have related pathophysiological processes (Maddison and Giorgini, 2015), and some of the disease-causing genes are overlapping (Drange et al., 2019). In our results, 16 of the 30 hub genes are related to AZ. Among them, AGT, APOE, GJA1, MAOA, RAB31, NOTCH2, PDLIM5, SLC1A3, SMAD2, and YAP1 are biomarkers or related risk factors of $\mathrm{AZ}$ (Wu et al., 2004; Ueberham et al., 2009; Kanai et al., 2013; Cheng et al., 2018; Kajiwara et al., 2018; Liang et al., 2018; Quartey et al., 2018; Xu et al., 2018; Zhao et al., 2018). This further proves that there may be a certain relationship between $\mathrm{BD}$ and $\mathrm{AZ}$, with overlapping mutant genes.
To the best of our knowledge, this is the first WGCNA analysis of RNA data from tissues of the prefrontal cortex of BD patients. The primary advantage of this study is to combine the gene expression data of $\mathrm{BD}$ patients with clinical traits. By choosing the appropriate weighting coefficient to weight the correlation coefficient between genes, the WGCNA algorithm could make the gene network obey the scale-free network distribution and divide the genes with similar expression into the same module. Then, the modules with high correlation with traits were selected, and hub genes in the modules were identified. Finally, the potential relationship between modules and hub genes and traits was further discussed through gene enrichment analysis.

Our research has some limitations. Compared to previous WGCNA analyses of other disease, our sample size was insufficient, and there might be some bias. Nevertheless, we were able to replicate many published findings successfully, and we used a separate database to verify the experimental results. Although most hub genes were verified, a few hub genes were not, probably because the samples of GSM12649 were not from the same platform. Another limitation is that similar studies have been published. However, we have discovered many new hub genes and enrichment pathways. Future studies will involve more experiments to prove and explain how hub genes and hub pathways affect $\mathrm{BD}$ development.

\section{CONCLUSION}

Our study based on WGCNA analyses found the 30 hub genes in three modules related to $\mathrm{BD}$, and the Hippo signaling pathway and positive regulation of transcription may be one of the potential BD mechanisms. These hub genes and enrichment pathways may have important clinical implications for $\mathrm{BD}$ treatment and diagnosis.

\section{DATA AVAILABILITY}

Publicly available datasets were analyzed in this study. This data can be found at: https://www.ncbi.nlm.nih.gov/geo/query/acc. cgi? acc $=$ GSE53987.

\section{AUTHOR CONTRIBUTIONS}

YL, CZ, and Y-MN conceived and designed the study. YL and CZ performed the analysis. YL, H-YG, and CZ analyzed the results. G-LG, JZ, and Y-MN contributed analysis tools. G-LG and CZ contributed to the writing of the manuscript. G-LG contributed to the revision of this research, especially in the design of "ranked at top $10 \%$ " and "GS $>0.2$, as the routine boundary value," and language modification. All authors reviewed the manuscript.

\section{SUPPLEMENTARY MATERIAL}

The Supplementary Material for this article can be found online at: https://www.frontiersin.org/articles/10.3389/fphys.2019. 01081/full\#supplementary-material 


\section{REFERENCES}

Adam, D. (2008). Trouble ahead for dementia care. Nurs. older people 20, 7-8. doi: $10.7748 /$ nop.20.6.7.s9

Arai, Y., and Taverna, E. (2017). Neural progenitor cell polarity and cortical development. Front. Cell. Neurosci. 11:384. doi: 10.3389/fncel.2017.00384

Arisi, I., D’Onofrio, M., Brandi, R., Felsani, A., Capsoni, S., Drovandi, G., et al. (2011). Gene expression biomarkers in the brain of a mouse model for Alzheimer's disease: mining of microarray data by logic classification and feature selection. J. Alzheimer's Dis. 24, 721-738. doi: 10.3233/JAD-2011101881

Barrett, T., Wilhite, S. E., Ledoux, P., Evangelista, C., Kim, I. F., Tomashevsky, M., et al. (2013). NCBI GEO: archive for functional genomics data sets-update. Nucleic Acids Res. 41, D991-D995. doi: 10.1093/nar/gks1193

Berridge, M. J. (2014). Calcium signalling and psychiatric disease: bipolar disorder and schizophrenia. Cell Tissue Res. 357, 477-492. doi: 10.1007/s00441-014$1806-\mathrm{z}$

Calkin, C., and Alda, M. (2012). Beyond the guidelines for bipolar disorder: practical issues in long-term treatment with lithium. Can. J. Psychiatry Rev. Can. Psychiatrie. 57, 437-445. doi: 10.1177/070674371205700707

Cardoso, T., Bauer, I. E., Meyer, T. D., Kapczinski, F., and Soares, J. C. (2015). Neuroprogression and cognitive functioning in bipolar disorder: a systematic review. Curr. Psychiatry Rep. 17:75. doi: 10.1007/s11920-015-0605-x

Chen, H. M., DeLong, C. J., Bame, M., Rajapakse, I., Herron, T. J., McInnis, M. G., et al. (2014). Transcripts involved in calcium signaling and telencephalic neuronal fate are altered in induced pluripotent stem cells from bipolar disorder patients. Transl. Psychiatry. 4:e375. doi: 10.1038/tp.2014.12

Chen, L., Yuan, L., Qian, K., Qian, G., Zhu, Y., Wu, C. L., et al. (2018). Identification of biomarkers associated with pathological stage and prognosis of clear cell renal cell carcinoma by Co-expression network analysis. Front. Physiol. 9:399. doi: 10.3389/fphys.2018.00399

Cheng, Z., Yin, J., Yuan, H., Jin, C., Zhang, F., Wang, Z., et al. (2018). Blood-derived plasma protein biomarkers for Alzheimer's disease in Han Chinese. Front. Aging Neurosci. 10:414. doi: 10.3389/fnagi.2018.00414

Ciani, L., and Salinas, P. C. (2005). WNTs in the vertebrate nervous system: from patterning to neuronal connectivity. Nat. Rev. Neurosci. 6, 351-362. doi: 10. $1038 /$ nrn 1665

Cox, J. H., Seri, S., and Cavanna, A. E. (2014). Clinical guidelines on long-term pharmacotherapy for bipolar disorder in children and adolescents. J. Clin. Med. 3, 135-143. doi: $10.3390 / \mathrm{jcm} 3010135$

De Bock, M., Kerrebrouck, M., Wang, N., and Leybaert, L. (2013). Neurological manifestations of oculodentodigital dysplasia: a Cx43 channelopathy of the central nervous system? Front. Pharmacol. 4:120. doi: 10.3389/fphar.2013. 00120

Drange, O. K., Smeland, O. B., Shadrin, A. A., Finseth, P. I., Witoelar, A., Frei, O., et al. (2019). Genetic overlap between Alzheimer's disease and bipolar disorder implicates the MARK2 and VAC14 genes. Front. Neurosci. 13:220. doi: $10.3389 /$ fnins. 2019.00220

Emoto, K. (2012). Signaling mechanisms that coordinate the development and maintenance of dendritic fields. Curr. Opin. Neurobiol 22, 805-811. doi: 10. 1016/j.conb.2012.04.005

Fabbri, C., and Serretti, A. (2016). Genetics of long-term treatment outcome in bipolar disorder. Prog. Neuro Psychopharmacol Biol. Psychiatry 65, 17-24. doi: 10.1016/j.pnpbp.2015.08.008

Farber, C. R. (2010). Identification of a gene module associated with BMD through the integration of network analysis and genome-wide association data. J. Bone Miner. Res. 25, 2359-2367. doi: 10.1002/jbmr.138

Faux, C. H., Turnley, A. M., Epa, R., Cappai, R., and Bartlett, P. F. (2001). Interactions between fibroblast growth factors and Notch regulate neuronal differentiation. J. Neurosci. 21, 5587-5596. doi: 10.1523/jneurosci.21-15-05587. 2001

Ferensztajn-Rochowiak, E., Tarnowski, M., Samochowiec, J., Michalak, M., Ratajczak, M. Z., and Rybakowski, J. K. (2016). Peripheral mRNA expression of pluripotency markers in bipolar disorder and the effect of long-term lithium treatment. Pharmacol. Rep. 68, 1042-1045. doi: 10.1016/j.pharep.2016. 06.006

Gonzalez, R. (2014). The relationship between bipolar disorder and biological rhythms. J. Clin. Psychiatry 75, e323-e331. doi: 10.4088/JCP.13r08507
Han, Y., Yu, H. X., Sun, M. L., Wang, Y., Xi, W., and Yu, Y. Q. (2014). Astrocyterestricted disruption of connexin-43 impairs neuronal plasticity in mouse barrel cortex. Eur. J. Neurosci. 39, 35-45. doi: 10.1111/ejn.12394

Hirschfeld, R. M. (2014). Differential diagnosis of bipolar disorder and major depressive disorder. J. Affect. Disord. 169(Suppl. 1), S12-S16.

Hogh, P. (2017). [Alzheimer's disease]. Ugeskrift for laeger 179:V09160686.

Irizarry, R. A., Hobbs, B., Collin, F., Beazer-Barclay, Y. D., Antonellis, K. J., Scherf, U., et al. (2003). Exploration, normalization, and summaries of high density oligonucleotide array probe level data. Biostatistics 4, 249-264. doi: 10.1093/ biostatistics/4.2.249

Iwamoto, K., Bundo, M., and Kato, T. (2005). Altered expression of mitochondriarelated genes in postmortem brains of patients with bipolar disorder or schizophrenia, as revealed by large-scale DNA microarray analysis. Hum. Mol. Genet. 14, 241-253. doi: 10.1093/hmg/ddi022

Kajiwara, Y., Wang, E., Wang, M., Sin, W. C., Brennand, K. J., Schadt, E., et al. (2018). GJA1 (connexin43) is a key regulator of Alzheimer's disease pathogenesis. Acta Neuropathol. Commun. 6:144. doi: 10.1186/s40478-0180642-X

Kanai, Y., Clemencon, B., Simonin, A., Leuenberger, M., Lochner, M., Weisstanner, M., et al. (2013). The SLC1 high-affinity glutamate and neutral amino acid transporter family. Mol. Aspects Med. 34, 108-120. doi: 10.1016/j.mam.2013. 01.001

Kiecker, C., and Niehrs, C. (2001). The role of prechordal mesendoderm in neural patterning. Curr. Opin. Neurobiol. 11, 27-33. doi: 10.1016/s0959-4388(00) 00170-7

Kim, A. H., Reimers, M., Maher, B., Williamson, V., McMichael, O., McClay, J. L., et al. (2010). MicroRNA expression profiling in the prefrontal cortex of individuals affected with schizophrenia and bipolar disorders. Schizophr. Res. 124, 183-191. doi: 10.1016/j.schres.2010.07.002

Kim, H. W., Rapoport, S. I., and Rao, J. S. (2010). Altered expression of apoptotic factors and synaptic markers in postmortem brain from bipolar disorder patients. Neurobiol. Dis. 37, 596-603. doi: 10.1016/j.nbd.2009.11.010

Kodaka, M., and Hata, Y. (2015). The mammalian Hippo pathway: regulation and function of YAP1 and TAZ. Cell. Mol. Life Sci. 72, 285-306. doi: 10.1007/s00018014-1742-9

Koike, T., Wakabayashi, T., Mori, T., Takamori, Y., Hirahara, Y., and Yamada, H. (2014). Sox2 in the adult rat sensory nervous system. Histochem. Cell Biol. 141, 301-309. doi: 10.1007/s00418-013-1158-x

Konopaske, G. T., Lange, N., Coyle, J. T., and Benes, F. M. (2014). Prefrontal cortical dendritic spine pathology in schizophrenia and bipolar disorder. JAMA Psychiatry. 71, 1323-1331.

Kuleshov, M. V., Jones, M. R., Rouillard, A. D., Fernandez, N. F., Duan, Q., Wang, Z., et al. (2016). Enrichr: a comprehensive gene set enrichment analysis web server 2016 update. Nucleic Acids Res. 44, W90-W97. doi: 10.1093/nar/gkw377

Langfelder, P., and Horvath, S. (2008). WGCNA: an R package for weighted correlation network analysis. BMC Bioinformatics 9:559. doi: 10.1186/14712105-9-559

Lanz, T. A., Reinhart, V., Sheehan, M. J., Rizzo, S. J. S., Bove, S. E., James, L. C., et al. (2019). Postmortem transcriptional profiling reveals widespread increase in inflammation in schizophrenia: a comparison of prefrontal cortex, striatum, and hippocampus among matched tetrads of controls with subjects diagnosed with schizophrenia, bipolar or major depressive disorder. Transl. Psychiatry. 9:151. doi: 10.1038/s41398-019-0492-8

Liang, J. W., Fang, Z. Y., Huang, Y., Liuyang, Z. Y., Zhang, X. L., Wang, J. L., et al. (2018). Application of weighted gene co-expression network analysis to explore the key genes in Alzheimer's disease. J. Alzheimer's Dis. 65, 1353-1364. doi: 10.3233/JAD- 180400

Liang, M. H., Wendland, J. R., and Chuang, D. M. (2008). Lithium inhibits Smad3/4 transactivation via increased CREB activity induced by enhanced PKA and AKT signaling. Mol. Cell. Neurosci. 37, 440-453. doi: 10.1016/j.mcn.2007.10.017

Liu, W. A., Chen, S., Li, Z., Lee, C. H., Mirzaa, G., Dobyns, W. B., et al. (2018), PARD3 dysfunction in conjunction with dynamic HIPPO signaling drives cortical enlargement with massive heterotopia. Genes Dev. 32, 763-780. doi: 10.1101/gad.313171.118

Liu, X., Hu, A. X., and Zhao, J. L. (2017). Identification of key gene modules in human osteosarcoma by co-expression analysis weighted gene co-expression network analysis (WGCNA). J. Cell. Biochem. 118, 3953-3959. doi: 10.1002/ jcb. 26050 
Liu, X., Zhang, T., He, S., Hong, B., Chen, Z., Peng, D., et al. (2014). Elevated serum levels of FGF-2, NGF and IGF-1 in patients with manic episode of bipolar disorder. Psychiatry Res. 218, 54-60. doi: 10.1016/j.psychres.2014.03.042

Machado-Vieira, R. (2018). Lithium, stress, and resilience in bipolar disorder: deciphering this key homeostatic synaptic plasticity regulator. J. Affect. Disord. 233, 92-99. doi: 10.1016/j.jad.2017.12.026

Maddison, D. C., and Giorgini, F. (2015). The kynurenine pathway and neurodegenerative disease. Semin. Cell Dev. Biol. 40, 134-141. doi: 10.1016/j. semcdb.2015.03.002

Medina, A., Burke, S., Thompson, R. C., Bunney, W. Jr., Myers, R. M., Schatzberg, A., et al. (2013). Glutamate transporters: a key piece in the glutamate puzzle of major depressive disorder. J. Psychiatr. Res. 47, 1150-1156. doi: 10.1016/j. jpsychires.2013.04.007

Meira-Lima, I. V., Pereira, A. C., Mota, G. F., Krieger, J. E., and Vallada, H. (2000). Angiotensinogen and angiotensin converting enzyme gene polymorphisms and the risk of bipolar affective disorder in humans. Neurosci. Lett. 293, 103-106. doi: 10.1016/s0304-3940(00)01512-3

Mizuno, T., Murakami, H., Fujii, M., Ishiguro, F., Tanaka, I., Kondo, Y., et al. (2012). YAP induces malignant mesothelioma cell proliferation by upregulating transcription of cell cycle-promoting genes. Oncogene 31, 5117-5122. doi: 10. 1038/onc. 2012.5

Moreau, M. P., Bruse, S. E., David-Rus, R., Buyske, S., and Brzustowicz, L. M. (2011). Altered microRNA expression profiles in postmortem brain samples from individuals with schizophrenia and bipolar disorder. Biol. Psychiatry 69, 188-193. doi: 10.1016/j.biopsych.2010.09.039

Mulligan, K. A., and Cheyette, B. N. (2017). Neurodevelopmental perspectives on Wnt signaling in psychiatry. Mol. Neuropsychiatry 2, 219-246. doi: 10.1159/ 000453266

Munkholm, K., Vinberg, M., and Kessing, L. V. (2016). Peripheral blood brainderived neurotrophic factor in bipolar disorder: a comprehensive systematic review and meta-analysis. Mol. Psychiatry 21, 216-228. doi: 10.1038/mp. 2015.54

O'Connell, K. S., McGregor, N. W., Lochner, C., Emsley, R., and Warnich, L. (2018). The genetic architecture of schizophrenia, bipolar disorder, obsessivecompulsive disorder and autism spectrum disorder. Mol. Cell. Neurosci. 88, 300-307. doi: 10.1016/j.mcn.2018.02.010

O'Shea, K. S., and McInnis, M. G. (2016). Neurodevelopmental origins of bipolar disorder: iPSC models. Mol. Cell. Neurosci. 73, 63-83. doi: 10.1016/j.mcn.2015. 11.006

Phi, J. H., Park, S. H., Paek, S. H., Kim, S. K., Lee, Y. J., Park, C. K., et al. (2007). Expression of Sox 2 in mature and immature teratomas of central nervous system. Mod. Pathol. 20, 742-748. doi: 10.1038/modpathol.3800793

Pinacho, R., Saia, G., Meana, J. J., Gill, G., and Ramos, B. (2015). Transcription factor SP4 phosphorylation is altered in the postmortem cerebellum of bipolar disorder and schizophrenia subjects. Eur. neuropsychopharmacol. 25, 16501660. doi: 10.1016/j.euroneuro.2015.05.006

Pompili, M., Gonda, X., Serafini, G., Innamorati, M., Sher, L., Amore, M., et al. (2013). Epidemiology of suicide in bipolar disorders: a systematic review of the literature. Bipolar Disord. 15, 457-490. doi: 10.1111/bdi.12087

Quartey, M. O., Nyarko, J. N. K., Pennington, P. R., Heistad, R. M., Klassen, P. C., Baker, G. B., et al. (2018). Alzheimer disease and selected risk factors disrupt a co-regulation of monoamine oxidase-A/B in the hippocampus, but not in the cortex. Front. Neurosci. 12:419. doi: 10.3389/fnins.2018. 00419

Raber, J., Huang, Y., and Ashford, J. W. (2004). ApoE genotype accounts for the vast majority of AD risk and AD pathology. Neurobiol. Aging 25, 641-650. doi: 10.1016/j.neurobiolaging.2003.12.023

Ravasz, E., Somera, A. L., Mongru, D. A., Oltvai, Z. N., and Barabasi, A. L. (2002). Hierarchical organization of modularity in metabolic networks. Science 297, 1551-1555. doi: 10.1126/science. 1073374

Sanches, M., Keshavan, M. S., Brambilla, P., and Soares, J. C. (2008). Neurodevelopmental basis of bipolar disorder: a critical appraisal. Prog. Neuro Psychopharmacol. Biol. Psychiatry 32, 1617-1627. doi: 10.1016/j.pnpbp.2008. 04.017

Shaltiel, G., Chen, G., and Manji, H. K. (2007). Neurotrophic signaling cascades in the pathophysiology and treatment of bipolar disorder. Curr. Opin. Pharmacol 7, 22-26. doi: 10.1016/j.coph.2006.07.005

Shannon, P., Markiel, A., Ozier, O., Baliga, N. S., Wang, J. T., Ramage, D., et al. (2003). Cytoscape: a software environment for integrated models of biomolecular interaction networks. Genome Res. 13, 2498-2504. doi: 10.1101/ gr.1239303

Smith, E. N., Bloss, C. S., Badner, J. A., Barrett, T., Belmonte, P. L., Berrettini, W., et al. (2009). Genome-wide association study of bipolar disorder in European American and African American individuals. Mol. Psychiatry 14, 755-763. doi: 10.1038/mp.2009.43

Stern, C. D. (2001). Initial patterning of the central nervous system: how many organizers? Nat. Rev. Neurosci. 2, 92-98. doi: 10.1038/35053563

Sundararajan, T., Manzardo, A. M., and Butler, M. G. (2018). Functional analysis of schizophrenia genes using GeneAnalytics program and integrated databases. Gene 641, 25-34. doi: 10.1016/j.gene.2017.10.035

Szklarczyk, D., Franceschini, A., Wyder, S., Forslund, K., Heller, D., Huerta-Cepas, J., et al. (2015). STRING v10: protein-protein interaction networks, integrated over the tree of life. Nucleic Acids Res. 43, D447-D452. doi: 10.1093/nar/ gku1003

Toker, L., Mancarci, B. O., Tripathy, S., and Pavlidis, P. (2018). Transcriptomic evidence for alterations in astrocytes and parvalbumin interneurons in subjects with bipolar disorder and schizophrenia. Biol. Psychiatry 84, 787-796. doi: 10.1016/j.biopsych.2018.07.010

Ueberham, U., Lange, P., Ueberham, E., Bruckner, M. K., Hartlage-Rubsamen, M., Pannicke, T., et al. (2009). Smad2 isoforms are differentially expressed during mouse brain development and aging. Int. J. Dev. Neurosci. 27, 501-510. doi: 10.1016/j.ijdevneu.2009.04.001

Uribe, E., and Wix, R. (2012). Neuronal migration, apoptosis and bipolar disorder. Rev. Psiquiatr. Salud Ment. 5, 127-133. doi: 10.1016/j.rpsm.2011.11.005

Vos, T., Flaxman, A. D., Naghavi, M., Lozano, R., Michaud, C., Ezzati, M., et al. (2012). Years lived with disability (YLDs) for 1160 sequelae of 289 diseases and injuries 1990-2010: a systematic analysis for the global burden of disease study 2010. Lancet 380, 2163-2196. doi: 10.1016/S0140-6736(12)61729-2

Woodbury, M. E., and Ikezu, T. (2014). Fibroblast growth factor-2 signaling in neurogenesis and neurodegeneration. J. Neuroimmune Pharmacol. 9, 92-101. doi: 10.1007/s11481-013-9501-5

Wu, M., Li, Y., Ji, C., Xu, J., Zheng, H., Zou, X., et al. (2004). Cloning and identification of a novel human gene PDLIM5, a homolog of AD-associated neuronal thread protein (AD7c-NTP). DNA seq. 15, 144-147. doi: 10.1080/ 10425170310001656756

Xu, M., Zhang, D. F., Luo, R., Wu, Y., Zhou, H., Kong, L. L., et al. (2018). A systematic integrated analysis of brain expression profiles reveals YAP1 and other prioritized hub genes as important upstream regulators in Alzheimer's disease. Alzheimer's Dement. 14, 215-229. doi: 10.1016/j.jalz.2017.08.012

Yin, L., Cai, Z., Zhu, B., and Xu, C. (2018). Identification of key pathways and genes in the dynamic progression of HCC based on WGCNA. Genes 9:E92.

Yip, A. M., and Horvath, S. (2007). Gene network interconnectedness and the generalized topological overlap measure. BMC Bioinformatics 8:22.

Zhang, B., and Horvath, S. (2005). A general framework for weighted gene co-expression network analysis. Stat. Appl. Genet. Mol. Biol. 4:17.

Zhang, L., Yue, T., and Jiang, J. (2009). Hippo signaling pathway and organ size control. Fly 3, 68-73. doi: 10.4161/fly.3.1.7788

Zhao, N., Liu, C. C., Qiao, W., and Bu, G. (2018). Apolipoprotein E, receptors, and modulation of Alzheimer's disease. Biol. Psychiatry 83, 347-357. doi: 10.1016/j. biopsych.2017.03.003

Zhu, L., Zhong, M., Elder, G. A., Sano, M., Holtzman, D. M., Gandy, S., et al. (2015). Phospholipid dysregulation contributes to ApoE4-associated cognitive deficits in Alzheimer's disease pathogenesis. Proc. Natl. Acad. Sci. U.S.A. 112, 11965-11970. doi: 10.1073/pnas.1510011112

Conflict of Interest Statement: The authors declare that the research was conducted in the absence of any commercial or financial relationships that could be construed as a potential conflict of interest.

The reviewer X-TZ declared a shared affiliation, with no collaboration, with one of the authors H-YG to the handling Editor at the time of review.

Copyright (C) $2019 \mathrm{Liu}, \mathrm{Gu}, \mathrm{Zhu}, \mathrm{Niu}$, Zhang and Guo. This is an open-access article distributed under the terms of the Creative Commons Attribution License (CC BY). The use, distribution or reproduction in other forums is permitted, provided the original author(s) and the copyright owner(s) are credited and that the original publication in this journal is cited, in accordance with accepted academic practice. No use, distribution or reproduction is permitted which does not comply with these terms. 